Open Access

\title{
Various autogenous fresh demineralized tooth forms for alveolar socket preservation in anterior tooth extraction sites: a series of 4 cases
}

Eun-Suk Kim ${ }^{1}$, In-Kyung Lee ${ }^{2}$, Ji-Yeon Kang ${ }^{3}$ and Eun-Young Lee ${ }^{4^{*}}$

\begin{abstract}
The aim of this study was to evaluate the clinical relevance of autogenous fresh demineralized tooth (Auto-FDT) prepared at chairside immediately after extraction for socket preservation. Teeth were processed to graft materials in block, chip, or powder types immediately after extraction. Extraction sockets were filled with these materials and dental implants were installed immediately or after a delay. A panoramic radiograph and a conebeam CT were taken. In two cases, tissue samples were taken for histologic examination.

Vertical and horizontal maintenance of alveolar sockets showed some variance depending on the Auto-FDT and barrier membrane types used. Radiographs showed good bony healing. Histologic sections showed that it guided good new bone formation and resorption pattern of the Auto-FDT.

This case series shows that Auto-FDT prepared at chairside could be a good material for the preservation of extraction sockets. This study will suggest the possibility of recycling autogenous tooth after immediate extraction.
\end{abstract}

Keywords: Alveolar socket preservation; Bone regeneration; Biocompatible; Bone substitute; Autogenous demineralized tooth

\section{Background}

Various methods and graft materials have been used to maintain alveolar ridge dimensions after tooth extraction, and these methods focus mainly on the preservation of hard tissue [1,2]. Recently, the use of tooth grafts has increased in alveolar bone defects $[3,4]$. Demineralized teeth can provide effective graft material [5]. However, immediate bone grafting after extraction used to be impossible because conventional decalcification takes three to five days. We adopted an optimized ultrasonic technology with periodic negative pressure and temperature control to enable the chairside preparation of tooth graft material. This approach dramatically reduced preparation time to $\leq 120$ min (block or chip type) and $40 \mathrm{~min}$ (powder type), while aseptic conditions

\footnotetext{
* Correspondence: ley926@chungbuk.ac.kr

${ }^{4}$ Deptartment of Oral and Maxillofacial Surgery, Chungbuk National University College of Medicine and Medical Research Institute, 52 Naesudong-ro, Heungduk-Gu, Cheongju, Chungbuk 361-763, Korea Full list of author information is available at the end of the article
}

were maintained in an isolated individual bioreactor tube system, this ability to prepare block or powder increases clinical availability [4]. Most importantly, tooth extraction and grafting for socket preservation can be performed on the same day [4].

The aim of this case report was to present the clinical usefulness of socket preservation using autogenous fresh demineralized tooth (Auto-FDT) prepared at chairside in anterior teeth extraction sites.

\section{Case presentation}

This study was approved by the Dankook University Jukjeon Dental Hospital institutional review board (2012004) and all participants signed an informed consent agreement (Table 1).

\section{Case 1}

Four anterior teeth (\#32-42) showed advanced periodontitis with mobility and severe alveolar resorption in a 74-

\section{勿 Springer}


Table 1 Summary of patients who underwent socket preservation using Auto-FDT

\begin{tabular}{|c|c|c|c|c|c|c|c|c|}
\hline No. & Age \& Gender & Extracted tooth number & Defect of alveolar bone & Sites of SP & Type of Auto-FDT & Membrane & Implant sites \& sizes (mm) & Delayed implant OP \\
\hline 1 & $74 / F$ & $31,32,41,42$ & Vertical \& horizontal & $31,32,41,42$ & Block & No & $32,42: 3.5 \times 13$ & Delayed: 3 months \\
\hline 2 & $43 / M$ & 11,21 & $\begin{array}{l}\text { Vertical \& horizontal } \\
\text { with labial plate } \\
\text { destruction }\end{array}$ & 11,21 & Block & Biosorb $^{\mathrm{TM}}$ : resorbable & $11: 4.0 \times 11.5,12: 3.5 \times 13$ & Delayed: 4 months \\
\hline 3 & $57 / M$ & 32,38 & $\begin{array}{l}\text { Vertical \& horizontal } \\
\text { with lingual plate } \\
\text { destruction }\end{array}$ & 31,32 & Chip & $\mathrm{CTi}$-mem ${ }^{\mathrm{TM}}$ : non-resorbable & $33,42: 3.5 \times 13$ & Simultaneous with SP \\
\hline 4 & $57 / M$ & $\begin{array}{l}11,12,21,2231,32, \\
41,42\end{array}$ & $\begin{array}{l}\text { Vertical \& horizontal } \\
\text { without labial plate } \\
\text { destruction }\end{array}$ & $\begin{array}{l}11,12,21,22, \\
31,32,41,42\end{array}$ & Powder & Colla tape: resorbable & $\begin{array}{l}12,22: 4.0 \times 11.5,32 \\
42: 3.5 \times 13\end{array}$ & Simultaneous with SP \\
\hline
\end{tabular}

SP socket preservation, Auto-FDT autogenous fresh demineralized tooth graft, $O P$ operation 


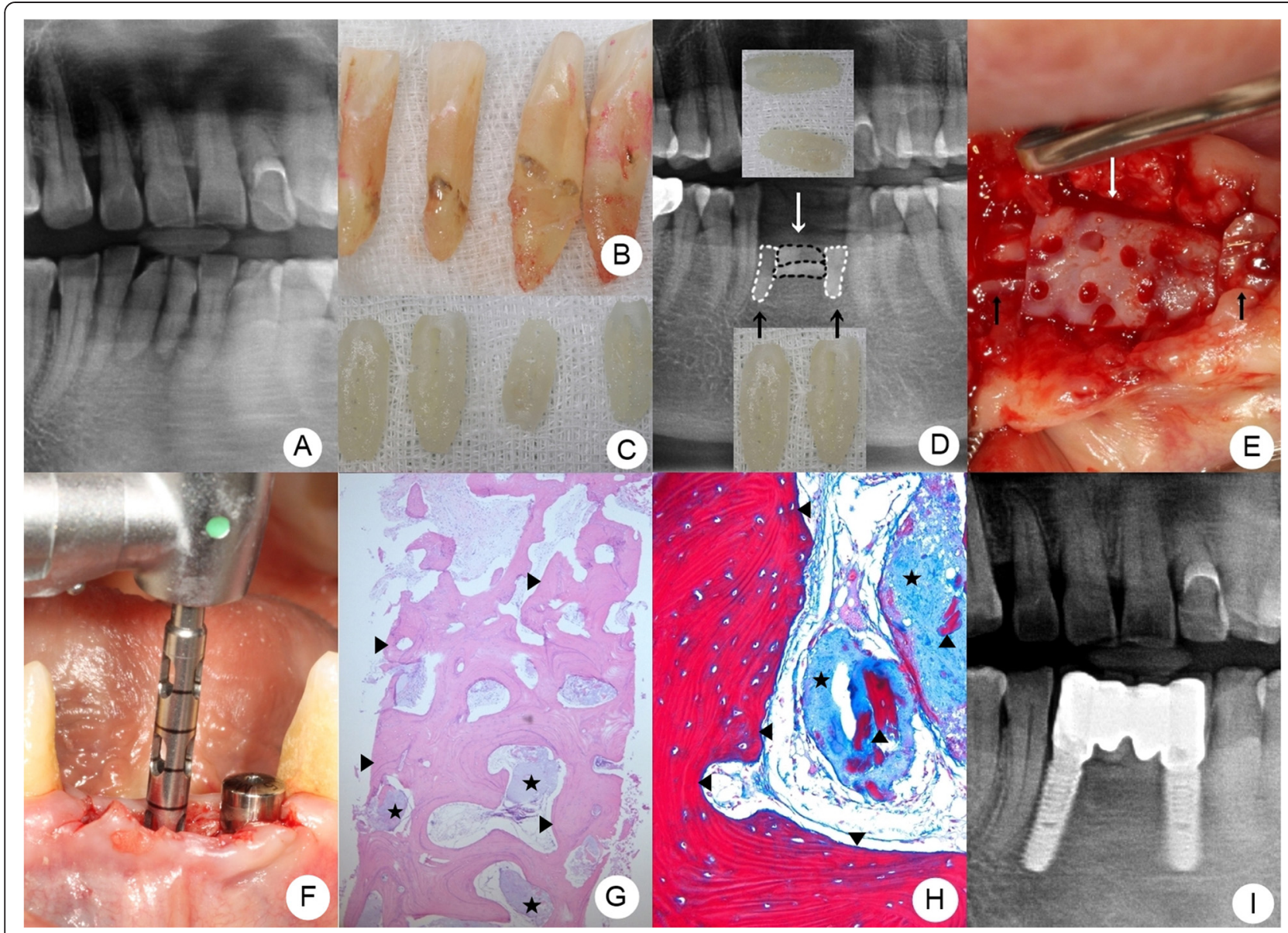

Fig. 1 Clinical and radiographic images of case 1. a Panoramic view before extraction of \# 31, 32, 41, 42. b Extracted lower anterior teeth. c Auto-FDT graft material (block type). d, e Blocks of Auto-FDT were inserted into the extraction socket vertically (black arrow) or horizontally (white arrow) depending on defect shape. $\mathbf{f}$ Bone core was taken at 3 months after the socket preservation. $\mathbf{g}$ Histologic section at postoperative 3 months showing that the Auto-FDT was almost completely replaced by new bone (H\&E, x40). $\mathbf{h}$ Higher magnification showing new bone in resorbable Auto-FDT (MT, x200). Auto-FDT (asterisk), new bone (black arrow head). i Panoramic view at 18 months after socket preservation surgery

year-old female (Fig. 1a). The extracted teeth were converted into block type Auto-FDT (Fig. 1b-e). No barrier membrane was used. After 3 months, graft sites were surgically reentered for implant placement. Socket preservation sites had maintained good, satisfactory bone and soft tissue contours for implant surgery despite slight horizontal resorption. Dental implants (TSIII CA, Osstem, Seoul, Korea) were placed in \#32, 42 sites and achieved initial stability with an insertion torque 20-30 Ncm. At 3 months after implant placement, 4unit fixed prosthesis was placed. A bone core was taken from the center of socket preservation sites and histologic sections were prepared at implant placement (Fig. 1f). It showed new bone around the grafted AutoFDT throughout the whole specimen. A wide range in the quantity of new bone formation was noted (Fig. 1gh). Good alveolar ridge height without bony resorption was achieved at 18 months after socket preservation surgery (Fig. 1i).

\section{Case 2}

A panoramic radiograph of a 43-year-old male revealed root fracture of \#11 and alveolar bone destruction around \#11, 21 (Fig. 2a). The blocks of Auto-FDT and collagen membrane (Biosorb ${ }^{\mathrm{Ts}}, 3 \mathrm{M}$ ESPE, USA) were used to fill sockets for implant placement after wound healing (Fig. 2b). At four months after socket preservation with block Auto-FDT, the graft was well consolidated but the amount of healed bone was slightly less than their initial quantities. Two implants were placed in \#11, 12 areas. At 4 months after implants placement, final restorations were completed. The patient was periodically recalled and followed up after prosthetic restoration for 19 months (Fig. 2c). No implants loss occurred, but more horizontal resorption was observed than in the other cases.

\section{Case 3}

A panoramic radiograph and conebeam CT of a 57year-old male showed an alveolar bone defect around 


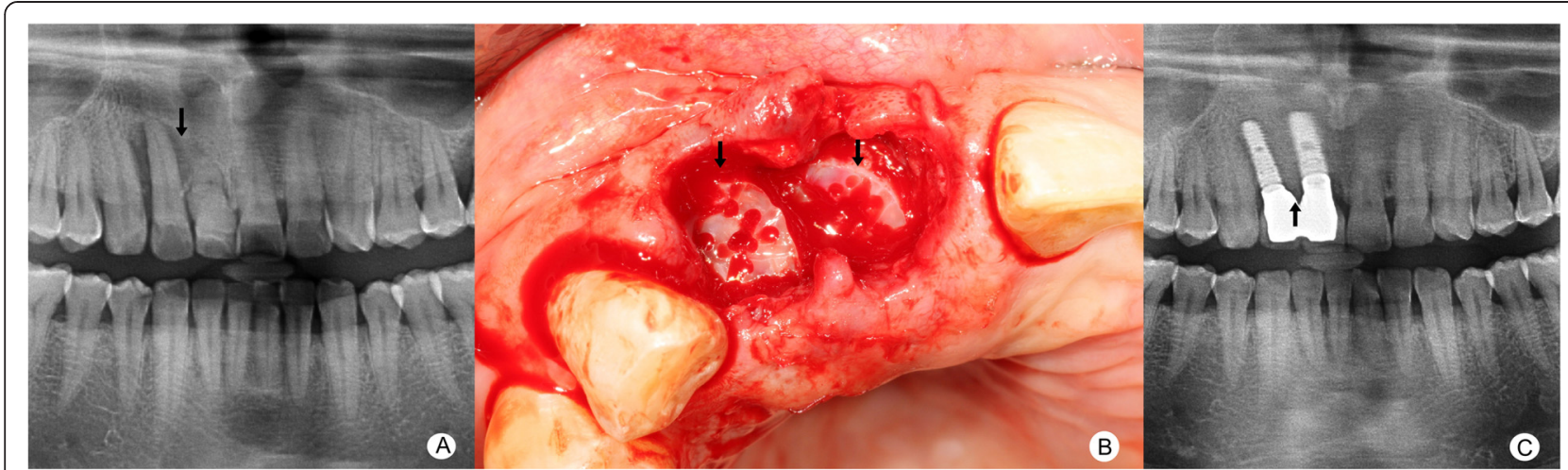

Fig. 2 Clinical and radiographic images of case 2. a Panoramic view before extraction of \#11, 12. A combined periodontic-endodontic lesion and root fracture of \#11 were diagnosed (black arrow). b Blocks of Auto-FDT (root portion, black arrow) were inserted into extraction sockets. c At 26 months after socket preservation, regenerated bone showed good maintenance between implants in panoramic view (black arrow)

\#32 including \#31 edentulous sites showing vertical and horizontal resorption with lingual plate destruction on lower incisors areas (Fig. 3a and b). Teeth (\#32, 38) were used to prepare chip Auto-FDT; prepared block AutoFDT within 120 min of extraction and then was changed to chip using a bone mill.

Extraction sockets of \#32 and the adjacent defect were filled with chip Auto-FDT. To prevent dissemination of particles and maintain of alveolar bone, a screw fixed thin titanium sheet $\left(\mathrm{CTi}-\mathrm{mem}^{\mathrm{Tx}}\right.$, Neobiotech, Seoul, Korea).
The patient was followed for 33 months after the socket preservation. The follow-up panoramic radiography showed a good alveolar ridge height without bony resorption (Fig. 3c).

A bone trephine bur of external diameter $2 \mathrm{~mm}$ was used to obtained a bone core from the centers of socket preservation sites at the uncovering surgery (Fig. 3d). In histologic sections, new bone (woven and lamella type) and a remnant of resorbed Auto-FDT were observed. Fibrous tissue and blood vessels were

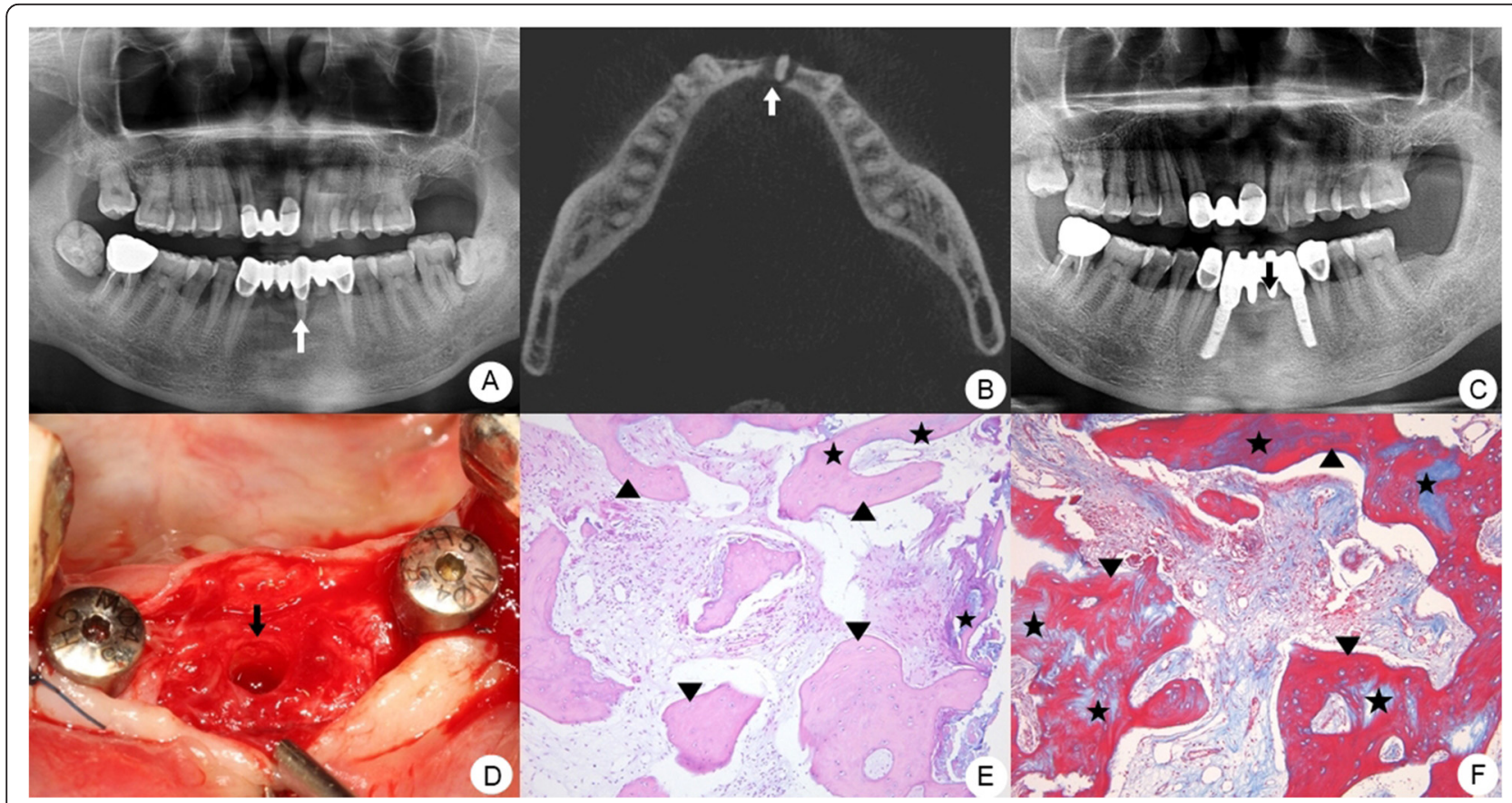

Fig. 3 Clinical, radiographic and histologic images of case 3. a Preoperative panoramic view (white arrow: bony defect). b Preoperative conebeam CT (white arrow: bony defect). c Panoramic view at 33 months after socket preservation (black arrow: alveolar crest). $\mathbf{d}$ A bone biopsy site in the center of a socket preservation site (black arrow: trephine drill hole). e Histologic section taken at 5 months after socket preservation showing remodeling of new bone around the Auto-FDT (H\&E, x100). f A Masson's trichrome stained section showing integration between newly formed bone and Auto-FDT (MT, x100). Auto-FDT (asterisk), new bone (black arrow head) 
also found (Fig. 3e). In the MT stained section, the interface between resorbed Auto-FDT and new bone was tight and interconnected (Fig. 3f).

\section{Case 4}

A 57-year-old male's panoramic radiograph revealed overall alveolar bone resorption (Fig. 4a). All anterior teeth (\#12-22 and \#32-42) were diagnosed as hopeless and extracted (Fig. 4b and c), and used to prepare powder Auto-FDT within 40 min of extraction.

We installed the implant installation (TSIII CA, Osstem, Seoul, Korea) in \#12, 22, 32, and 42 with socket preservation with powder Auto-FDT (Fig. 4d). To prevent dissemination of Auto-FDT particles, a collagen sheet (Colla tape, Zimmer, Germany) covered extraction sockets (Fig. 4e and f). The healed soft tissues were observed at 2 weeks after surgery (Fig. $4 \mathrm{~g}$ and $\mathrm{h}$ ).

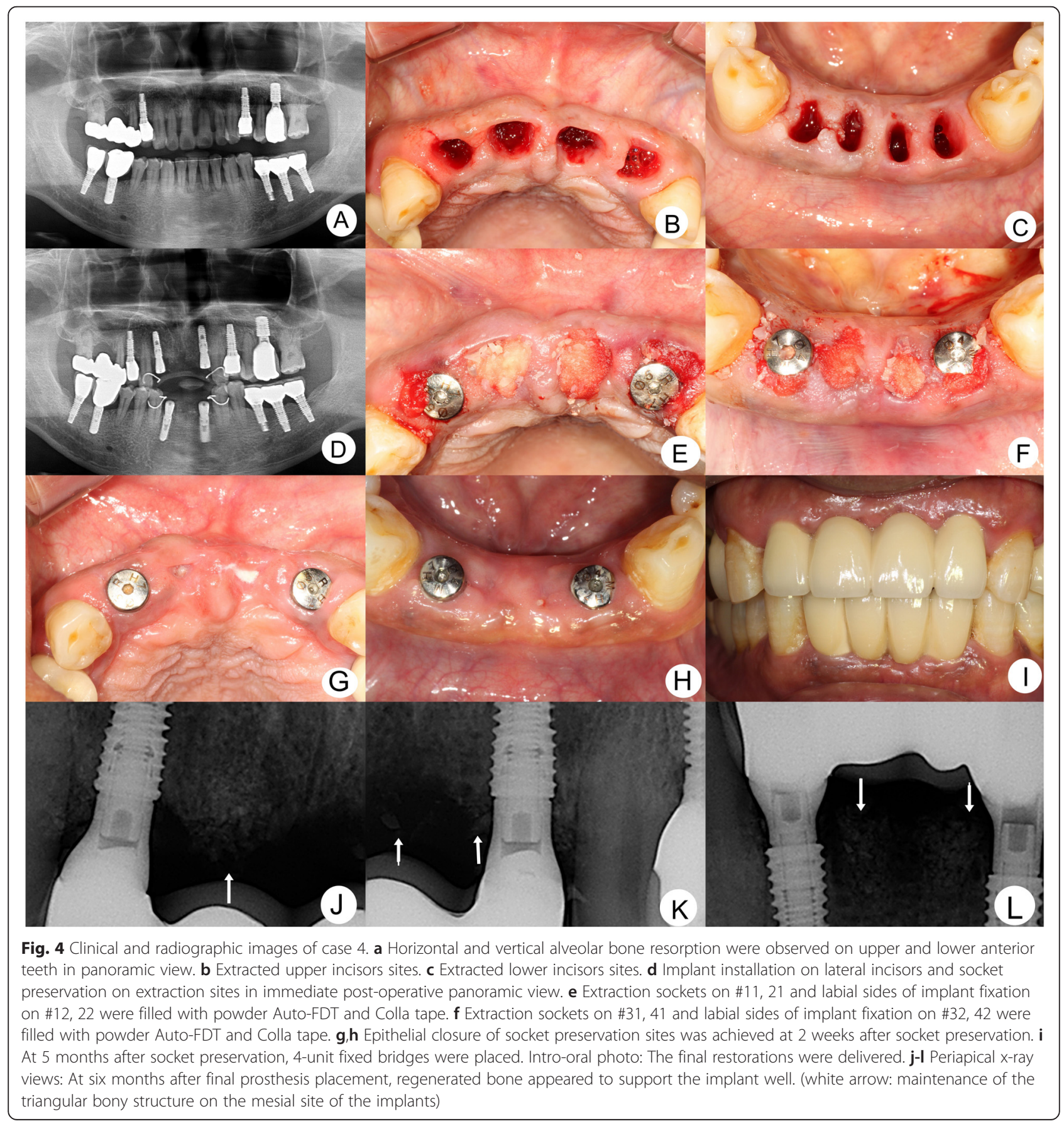


After a 4-month healing period, 4-unit fixed bridges were placed in the upper and lower sites for implant installation respectively (Fig. 4i). Clinical and radiological examinations at 6 months after prosthesis placement showed horizontal and vertical volumes of extraction sockets were well maintained (Fig. 4j-l).

\section{Discussion}

Tooth extraction is invariably followed by loss of height and width of the alveolar process. During natural healing after extraction, reductions in width of between 2.6 and $4.6 \mathrm{~mm}$ and in height of between 0.4 and $3.9 \mathrm{~mm}$ are observed [6], and these result in narrowing and shortening of the residual ridge [7]. Osseous augmentation procedures for creating bone volume for dental implants often involve the use of grafting materials with or without barrier membranes $[1,2,8]$.

Tooth is a potentially valuable graft material, and demineralized dentin has recently been used to reconstruct alveolar bone $[3,4]$. However, the long processing time required to demineralize and prepare teeth for socket preservation is problematic. Lee et al.[4] reported a new method to reduce processing times chairside, and reduced the whole preparation process of block or chip Auto-FDT to less than $120 \mathrm{~min}$. It is possible to commence socket preservation immediately after extraction using the extracted tooth. Auto-FDT is an organic graft material that has been chemically treated to remove its inorganic components. The calcium (inorganic component) concentration of block Auto-FDT is 6.5 wt.\% whereas that of normal dentin is $31 \mathrm{wt} . \%$ at a depth of $300-600 \mu \mathrm{m}$ from external surfaces, indicating that $79 \%$ of calcium is removed [4]. The remaining inorganic and organic components are needed in grafted areas for bone formation by osteoinduction or osteoconduction [6]. It is possible to control levels of inorganic components by changing tooth processing times.

The results of clinical and radiologic studies performed in the present study suggest that socket dimensional changes following tooth extraction are prevented or reduced using Auto-FDT. Radiographs obtained at 33 months after socket preservations revealed maintenance of alveolar ridges in case 3. In the patients treated with block Auto-FDT with or without resorbable membrane such as case 1 and 2, a slight width reduction was observed during clinical examinations, whereas heights were maintained. The reasons for resorption were that block Auto-FDT was placed with natural root size and shape into extracted sites without overcorrection and space maintained improperly with or without resorbable membrane. It was not enough to prevent the unfavorable stress of lip movement and soft tissue contracture without stress-shielding barrier membrane.
Chip Auto-FDT with a stress-shielding barrier membrane (titanium sheet) for space maintenance (case 3), it improved ridge height and width dimensions when compared to block type with/ without resorbable membrane (case 1 and 2), and it was possible to maintain of a space for graft materials using titanium sheet to prevent unfavorable stresses.

Powder Auto-FDT and chip or block Auto-FDT with a stress-shielding barrier membrane might preserve extraction sockets better with respect to height and width than the block Auto-FDT alone as used in the present study. In case 4, power Auto-FDT valuably preserved as determined by clinical and radiologic findings without dimensional changes. Periapical radiograms showed good marginal bone response and the absence of any residual vertical bone defect (Fig. 4j-1). Furthermore, the triangular shape of bone in the mesial side of the implant was maintained. The powder type is believed to be resorbed more slowly than the other two types, as the time of demineralization is less than that of the block type, which makes it possible to maintain a space for bone formation and to prevent unfavorable stress without a titanium sheet.

Several limitations of this study should be considered. In particular, it was not a prospective study and measurements of alveolar ridge dimensions were not performed. Nevertheless, it presents good results for socket preservation using Auto-FDT with respect to implant installation and alveolar ridge maintenance.

\section{Conclusions}

Socket preservation using powder, chip or block type Auto-FDT with a stress-shielding barrier membrane was effective in maintaining ridge heights and widths for implants. Further studies are needed to determine the effects of different types of bony defect on the functions of Auto-FDT and barrier membranes.

\section{Competing interest}

The authors declare that they have no competing interests.

\section{Authors' contributions}

ESK has made substantial contributions in reviewing articles, interpreting the data, and drafting the manuscript. IKL has made substantial contributions in revising the manuscript critically for important intellectual content. JYK has made substantial contributions to conception and design. EYL conceived of the study, and participated in its design and coordination and has given final approval of the version to be published. All authors read and approved the final manuscript.

\section{Acknowledgement}

This work was supported by a research grant from Chungbuk National University Hospital in 2015.

\section{Author details}

${ }^{1}$ Department of Oral and Maxillofacial Surgery, Weerae Dental Clinics, Seoul, South Korea. ${ }^{2}$ Department of Periodontology, Jukjeon Dental Hospital, Dankook University College of Dentistry, Yongin, Korea. ${ }^{3}$ Department of Oral and Maxillofacial Surgery, Dongtan Sacred Heart Hospital, Hallym University, 
Hwaseong, Korea. ${ }^{4}$ Deptartment of Oral and Maxillofacial Surgery, Chungbuk National University College of Medicine and Medical Research Institute, 52 Naesudong-ro, Heungduk-Gu, Cheongju, Chungbuk 361-763, Korea.

Received: 23 July 2015 Accepted: 19 August 2015

Published online: 03 September 2015

\section{References}

1. Lekovic V, Kenney E, Weinlaender M, Han T, Klokkevold P, Nedic M, Orsini M. (1997) A bone regenerative approach to alveolar ridge maintenance following tooth extractions. Report of 10 cases. J Periodontol 68:563-570

2. Carmagnola D, Adriaens P, Berglundh T (2003) Healing of human extraction sockets filled with Bio-Oss. Clin Oral Implants Res 14:137-143

3. Lee JY, Lee JH, Kim YK (2013) Comparative analysis of guided bone regeneration using autogenous tooth bone graft material with and without resorbabe membrane. J Dent Sci 8:281-286

4. Lee EY, Kim EK, Kim KW (2014) SEM and EDS studies on processed tooth graft material by vacuum-ultrasonic acceleration. Maxillofac Plast Reconstr Surg 36:103-110

5. Yeomans JD, Urist MR (1967) Bone induction by decalcified dentine implanted into oral, osseous and muscles tissues. Arch Oral Biol 12:999-1008

6. Ten Heggeler JMAG, Slot DE, Van der Wijden GA (2011) Effect of socket preservation therapies following tooth extraction in non-molar regions in humans: a systematic review. Clin Oral Implants Res 23:779-788

7. Pinho MN, Roriz VL, Novaes ABJ, Taba M Jr, Grisi MF, de Souza SL, Palioto DB. (2006) Titanium membranes in prevention of alveolar collapse after tooth extraction. Implant Dent 15:53-61

8. Lekovic V, Carmargo P, Klokkevold P, Weinlaender M, Kenney E, Dimitrijevic B, Nedic M. (1998) Preservation of alveolar bone in extraction sockets using bioabsorbable membranes. J Periodontol 69:1044-1049

\section{Submit your manuscript to a SpringerOpen ${ }^{\circ}$ journal and benefit from:}

- Convenient online submission

- Rigorous peer review

- Immediate publication on acceptance

- Open access: articles freely available online

- High visibility within the field

- Retaining the copyright to your article

Submit your next manuscript at springeropen.com 